\title{
EXTENDING REAL MAPS DEFINED ON A SUBSET OF A DISK
}

\author{
B. J. BALL, JO FORD AND E. S. THOMAS, JR. ${ }^{1}$
}

Let $K$ and $Q$ denote, respectively, the unit circular disk and the unit square disk in $E^{2}$. Let $\Delta: K \rightarrow[0,1]$ and $\pi: Q \rightarrow[0,1]$ be defined by $\Delta(x, y)=\left(x^{2}+y^{2}\right)^{1 / 2}$ and $\pi(x, y)=x$. Suppose $T$ is a subset of a disk $D$ and $f$ is a mapping of $T$ into $[0,1]$; we are concerned here with conditions under which $f$ can be extended to a mapping of $D$ onto $[0,1]$ which is topologically equivalent to $\Delta$ or to $\pi$. Recall that a mapping $u: A \rightarrow B$ is topologically equivalent to a mapping $w: A^{\prime} \rightarrow B^{\prime}$ if there exist homeomorphisms $h: A \rightarrow A^{\prime}$ and $k: B \rightarrow B^{\prime}$ such that $k u=w h$.

It was shown in [2] that if $T$ is a compact 0 -dimensional subset of Int $D$, then any mapping $f$ of $T$ into the open interval $(0,1)$ has an extension to a mapping of $D$ onto $[0,1]$ which is topologically equivalent to $\pi$ (and hence also an extension equivalent to $\Delta$ ). However, the existence of a single arc in $T$, even though this arc is a subset of a point-inverse of $f$, may make the desired extension impossible. For example, if $T$ is a subset of Int $D$ consisting of a horizontal interval $A$ together with points $x_{1}, x_{2}, x_{3}, \cdots$ such that the sequence $\left\{x_{2 n}\right\}$ converges to a midpoint of $A$ from above and $\left\{x_{2 n+1}\right\}$ converges to a midpoint of $A$ from below, then the mapping $f$ defined by $f(A)=1 / 2$, $f\left(x_{n}\right)=1 / 2+1 / 3 n$ cannot be extended to a mapping equivalent to $\pi$. We therefore restrict attention in this paper to the case in which the common part of $T$ and the interior of $D$ is totally disconnected.

First we show that if $T$ is a compact subset of a disk $D$ whose intersection with the interior of $D$ is totally disconnected and $T$ does not contain the boundary of $D$, then any mapping $f$ of $T$ into $[1 / 2,1]$ such that $T \cap \operatorname{Bd} D=f^{-1}(1)$ can be extended to a mapping of $D$ onto $[0,1]$ which is topologically equivalent to $\pi$. Using this result, we show that if $T$ is a compact subset of a disk $D$ whose intersection with the interior of $D$ is totally disconnected and $T$ contains the boundary of $D$, then any mapping $f$ of $T$ into $[1 / 2,1]$ such that $\mathrm{Bd} D=f^{-1}(1)$ can be extended to a mapping of $D$ onto $[0,1]$ which is topologically equivalent to $\Delta$.

It follows as a special case of results in [1] that these theorems do not directly generalize to higher dimensions. If $n \geqq 3,1 \leqq k \leqq n$, and

\footnotetext{
Received by the editors December 30, 1966 and, in revised form, September 18, 1967.

1 Research supported by NSF grant GP-5935.
} 
$\pi^{\prime}$ denotes the projection onto the last $k$ coordinates of points of $I^{n}$, there is a Cantor set $A$ in Int $I^{n}$ such that Int $I^{n}-A$ is not simply connected. If $f$ is the constant map from $A$ to $p=(1 / 2,1 / 2, \cdots$, $1 / 2)$ in Int $I^{k}$, then there is no extension of $f$ to all of $I^{n}$ that is equivalent to $\pi^{\prime}$. To see this, suppose that $f^{\prime}$ is a mapping from $I^{n}$ onto $I^{k}$ extending $f$ for which there are self homeomorphisms $h$ and $g$ of $I^{n}$ and $I^{k}$ respectively such that $g f^{\prime}=\pi^{\prime} h$. Then $h(A)$ would lie in $\pi^{\prime-1}(g(p))$, and if $q$ denotes the projection given by $q\left(x_{1}, x_{2}, \cdots, x_{n}\right)$ $=\left(x_{2}, x_{3}, \cdots, x_{n}\right)$ in $I^{n-1}$, then $q h(A)$ is a Cantor set in Int $I^{n-1}$, and by Theorem $3 \mathrm{E}$ of [1], Int $I^{n}-h(A)$ is simply connected-a contradiction.

The following lemmas are used frequently in the construction which follow and so are stated separately.

LEMMA 1. If $S$ is a compact 0-dimensional subset of $E^{2}$ covered by a collection $\mathrm{G}$ of open sets, then there is a refinement of $\mathrm{G}$ of mutually exclusive disks whose interiors cover $S$.

This follows readily from the fact that every Cantor set in $E^{2}$ is tame.

Definition. The arc (or disk) $A$ spans the connected domain $G$ from $x$ to $y$ means that $x$ and $y$ are two boundary points of $G, A$ $-\{x, y\}$ is a connected subset of $G$, and $A \cap \operatorname{Bd} G=\{x, y\}$.

Lemma 2. If $T$ is a compact subset of $Q$ such that $T \cap$ Int $Q$ is 0 -dimensional and $D$ is a disk in $Q$ with boundary points $x$ and $y$, then there exists a disk which spans Int $D$ from $x$ to $y$ which does not intersect $T-\{x, y\}$.

Indication of Proof. It is not difficult to construct an arc $A$ spanning Int $D$ from $x$ to $y$ and missing $T-\{x, y\}$. If $A$ is such an arc, there is a disk $D^{\prime}$ containing $A$ such that $D^{\prime}-\{x, y\}$ is a subset of (Int $D)-T$, and hence $D^{\prime}$ spans Int $D$ from $x$ to $y$.

THEOREM 1. If $T$ is a compact subset of a disk $D, \mathrm{Bd} D \nsubseteq T$, and $T \cap$ Int $D$ is totally disconnected, then every mapping of $T$ into $[1 / 2,1]$ whose inverse of 1 is $T \cap \mathrm{Bd} D$ can be extended to a mapping of $D$ onto $[0,1]$ which is equivalent to $\pi$.

Proof. If $T$ and $D$ are as in the hypothesis, then there is a self homeomorphism $v$ of $E^{2}$ such that $v(D)$ is $Q$ and $V(T \cap \mathrm{Bd} D)$ is contained in the right vertical edge $A=\{(1, y) \mid 0 \leqq y \leqq 1\}$. Let $T^{\prime}$ denote $A \cup_{v}(T)$, and suppose $f$ is a mapping of $T$ into $[1 / 2,1]$ such that $f^{-1}(1)=T \cap \operatorname{Bd} D$. It will be shown that there exists a self homeo- 
morphism $w$ of $Q$ such that for each $t$ in $T^{\prime}, \pi w(t)=f\left(v^{-1}(t)\right)$. From this it will follow that $\pi w v$ is an extension of $f$ that is equivalent to $\pi$. Denote by $f^{\prime}$ the composition $f v^{-1}$.

There is a disk $D_{1}$ spanning Int $Q$ from $(0,1 / 2)$ to $(1,1 / 2)$ which contains only the point $(1,1 / 2)$ of $T^{\prime}$; and there is an arc $L_{1}$ spanning Int $Q$ from $(3 / 4,0)$ to $(3 / 4,1)$ which misses $T^{\prime}$, intersects the boundary of $D_{1}$ in exactly two points, and lies entirely to the right of the vertical line through $(1 / 2,0)$. Clearly $L_{1}$ divides $Q$ into two disks; denote the one containing $A$ by $L_{1}^{\prime}$, and if $E_{1}$ denotes $\left(Q-\operatorname{Int} D_{1}\right)$ $\cap L_{1}^{\prime}$, then $E_{1}$ contains $T^{\prime} \cap L_{1}^{\prime}, \mathrm{Bd} E_{1} \cap T^{\prime}=A$, the closure of each component of Int $E_{1}$ is a disk of diameter less than 1 , and $A$ is accessible from the domain Int $Q-E_{1}$.

Recall that "the point set $A$ is accessible from the domain $G$ " means that for some point $x$ in $A$, there is an arc in $G \cup\{x\}$ which contains $x$.

There are disks $D_{2}$ and $D_{3}$ such that (1) $D_{2}$ spans the lower component of Int $E_{1}$ from a point of $L_{1}$ to $(1,1 / 4)$ and $D_{3}$ spans the upper component from a point of $L_{1}$ to $(1,3 / 4)$, and (2) $D_{2}$ contains only the point $(1,1 / 4)$ of $T^{\prime}$ and $D_{3}$ the point $(1,3 / 4)$ of $T^{\prime}$. The boundary of $D_{1} \cup D_{2} \cup D_{3}$ consists of two arcs emanating from $(1,1 / 4)$ plus two arcs from $(1,1 / 2)$ plus two from $(1,3 / 4)$. Hence there is a vertical line $V$ between $L_{1}$ and $A$ such that each component of Int $Q$ $-\left(V \cup \operatorname{Bd}\left(D_{1} \cup D_{2} \cup D_{3}\right)\right)$ to the right of $V$ has diameter less than $1 / 2$. There is an arc $L_{2}$ spanning Int $Q$ from top to bottom between $V$ and $A$ that misses $T^{\prime}$ and intersects each of $\operatorname{Bd} D_{1}, \operatorname{Bd} D_{2}$ and $\operatorname{Bd} D_{3}$ in exactly two points, and such that $L_{2}$ lies to the right of the vertical line through $(3 / 4,0)$. Again, $L_{2}$ divides $Q$ into two cells, $L_{2}^{\prime}$ denotes the one containing $A$, and if $E_{2}$ denotes $\left[E_{1}-\operatorname{Int}\left(D_{2} \cup D_{3}\right)\right] \cap L_{2}^{\prime}$, then $E_{2} \subset E_{1}, E_{2}$ contains $T^{\prime} \cap L_{2}^{\prime}, \mathrm{Bd} E_{2} \cap T^{\prime}=A, A$ is accessible from each of the two components of (Int $\left.E_{1}\right)-E_{2}$, and the closure of each component of Int $E_{2}$ is a disk of diameter less than $1 / 2$.

In this way, a sequence $\left\{E_{i}\right\}$ is constructed such that for each $i$ :

(1) $E_{i}$ is the union of $2^{i}$ disks each of which has diameter less than $1 / 2^{i-1}$ and intersects $A$ in an arc, such that if two of these disks intersect, then the intersection is a single point of $A$,

(2) $\left(\operatorname{Bd} E_{i}\right) \cap T^{\prime}=A$,

(3) $E_{i} \subset E_{i-1}$

(4) there is an arc spanning Int $Q$ between the vertical line through $\left(1-1 / 2^{i+1}, 0\right)$ and $A$ such that $E_{i}$ contains in its interior every point of $T^{\prime}$ to the right of this arc, and

(5) if $i>j \geqq 0$, then $A$ is accessible from each of the $2^{j}$ components of (Int $\left.E_{j}\right)-E_{i}$. 
For each $i$, denote by $H_{i}$ the collection of closures of components of Int $E_{i}$. Let $G_{1}$ denote a finite collection of mutually exclusive disks in (Int $Q)-E_{i}$ whose interiors cover $T^{\prime}-E_{1}$. If $G_{i}$ has been defined, define $G_{i+1}$ to be a collection of mutually exclusive disks in (Int $Q$ ) $-E_{i+1}$ whose interiors cover $T^{\prime}-E_{i+1}$ such that each disk has diameter less than $1 / 2^{i+1}$ and lies in the interior of some element of $G_{i} \cup H_{i}$.

Since $f^{\prime}$ is a continuous mapping on the compact set $T$ and mesh $G_{i} \cup H_{i} \rightarrow 0$ as $i \rightarrow \infty$, for each $\epsilon>0$ there is an integer $n$ such that $f^{\prime}\left(k \cap T^{\prime}\right)<\epsilon$ for each $k \in G_{n} \cup H_{n}$. Hence it may be assumed that for each $n$, the diameter of $f^{\prime}\left(k \cap T^{\prime}\right)$ is less than $1 / 2^{n+1}$ if $k$ is in $G_{n} \cup H_{n}$.

There is a self homeomorphism $u_{1}$ of $Q$ such that

(1) if $c \in G_{1}$, then $u_{1}(c)$ is a rectangular disk of diameter less than $1 / 2$ with sides parallel to the coordinate axes,

(2) if $b \in H_{1}$, then $u_{1}(b)$ is a quadrilateral disk of diameter less than $1 / 2$,

(3) if $c \in G_{1} \cup H_{1}$, then $\pi$ (Int $\left.u_{1}(c)\right)$ contains $f^{\prime}\left(c \cap T^{\prime}\right)$, and

(4) $u_{1}$ is the identity on $\operatorname{Bd} Q$.

Suppose for each $i<m, u_{i}$ has been defined. Let $w_{m}$ denote the composition $u_{m-1} \cdot u_{m-2} \cdots u_{2} \cdot u_{1}$. There is a self homeomorphism $u_{m}$ of $Q$ such that

(1) if $c \in G_{m}$, then $u_{m} w_{m}(c)$ is a rectangular disk of diameter less than $1 / 2^{m}$ with sides parallel to the coordinate axes,

(2) if $b \in H_{m}$, then $u_{m} w_{m}(b)$ is the interior of a quadrilateral disk of diameter less then $1 / 2^{m}$,

(3) if $c \in G_{m} \cup H_{m}$, then $\pi$ (Int $\left.u_{m} w_{m}(c)\right)$ contains $f^{\prime}\left(c \cap T^{\prime}\right)$, and

(4) $u_{m}$ is the identity on $\mathrm{Bd} Q \cup\left(Q-\cup\left\{w_{m}(c) \mid c \in G_{m-1} \cup H_{m-1}\right\}\right)$.

This defines the sequence $\left\{w_{i}\right\}$ of self homeomorphisms of $Q$. Since $u_{i}$ moves no point more than $1 / 2^{i-1},\left\{w_{i}\right\}$ is Cauchy and converges to a continuous function $w$ of $Q$ onto $Q$. By condition (3) on each $u_{i}$, $\pi w(x)=f^{\prime}(x)$ for all $x$ in $T^{\prime}$. If there are an $x$ and $y$ in $Q$ such that $w(x)=w(y)$, then for each $i, w_{i}(x)$ and $w_{i}(y)$ must be (if unequal) in the same quadrilateral disk or rectangular disk, as described in properties (2) and (1) of $u_{i}$. Hence $w_{i+1}^{-1}(x)$ and $w_{i+1}^{-1}(y)$ are both in the same element of $G_{i} \cup H_{i}$. Hence, $d(x, y) \leqq 1 / 2^{i}$ so that $x=y$. Hence $w$ is a homeomorphism and since $\pi w(t)=f^{\prime}(t)=f v^{-1}(t)$ for $t \in T^{\prime}$, w has the required properties. Because it is needed in the proof of Theorem 2, note that $w(x)=x$ for all $x \in \mathrm{Bd} Q$.

THEOREM 2. If $T$ is a compact subset of a disk $D$ such that $\mathrm{Bd} D \subset T$ and $T \cap I n t D$ is totally disconnected, then every map from $T$ into $[1 / 2,1]$ whose inverse of 1 is $\mathrm{Bd} D$ can be extended to a mapping of $D$ onto $[0,1]$ which is equivalent to $\Delta$. 
Proof. As in the proof of Theorem 1, there is no loss in generality if $D$ is assumed to be the unit circular disk centered at the origin. Also, assume that the circular disk $D^{\prime}$ centered at the origin with radius $1 / 3$ contains no point of $T$. Let $B_{1}$ and $B_{2}$ be disjoint arcs irreducible from $\operatorname{Bd} D^{\prime}$ to $\operatorname{Bd} D$ that do not intersect $T \cap \operatorname{Int} D$. There is a homeomorphism of $D$ onto itself that takes $B_{1} \cup B_{2}$ onto two straight line segments each a subset of a radial line in $D$, so there is no loss in generality in assuming that $B_{1}$ and $B_{2}$ are themselves each a subset of a radial line in $D$. If $D_{1}$ and $D_{2}$ are the disks into which $D-$ Int $D^{\prime}$ is divided by $B_{1} \cup B_{2}$, it follows from Theorem 1 that there exist homeomorphisms $u_{1}$ of $D_{1}$ onto itself and $u_{2}$ of $D_{2}$ onto itself such that for $i=1,2$,

(1) $u_{i}$ is the identity on $\operatorname{Bd} D_{i}$ and

(2) for each $x$ in $[1 / 3,1), u_{i}\left(f^{-1}(x) \cap D_{i}\right)$ is a subset of the circle with center $(0,0)$ and radius $x$. There is a homeomorphism $u$ of $D$ on to itself which is an extension of $u_{1}$ and $u_{2}$ and is the identity on $D^{\prime}$. The mapping $\Delta u$ is an extension of $f$ which is equivalent to $\Delta$.

Theorem 2 may be applied to give results on the problem of embedding collections of compact, totally disconnected subsets of the plane in collections of arcs or simple closed curves. One such theorem is the following.

Corollary. Suppose $M_{1}, M_{2}, M_{3}, \cdots$ is a sequence of compact, totally disconnected subsets of $E^{2}$ converging to a nondegenerate compact set $M$ which does not intersect $U M_{i}$. Then there is a homeomorphism of $E^{2}$ onto itself which takes each $M_{i}$ into a different circle with center $(0,0)$ if and only if $M$ is a subset of a simple closed curve which does not intersect $U M_{i}$ and does not separate two points of any $M_{i}$, and there is a homeomorphism of $E^{2}$ onto itself which takes each $M_{i}$ into a different horizontal interval if and only if $M$ is a proper subset of such a simple closed curve.

Proof. The necessity of each condition is clear.

Suppose $M$ is a subset of a simple closed curve $C$ and that each $M_{i}$ is a subset either of Int $C$ or of Ext $C$. Assume that $C$ is the unit circle, and let $I$ and $E$ denote, respectively, the interior and the exterior of $C$.

It follows from Theorem 2 that there is a homeomorphism $h_{1}$ of the plane onto itself which is the identity on $E$ and which takes each $M_{i}$ lying in $I$ into a circle with center $(0,0)$. Applying Theorem 2 to the one-point compactification of $E$, it can be shown that there is a homeomorphism $h_{2}$ of the plane onto itself which is the identity on $I$ and which takes each $M_{i}$ lying in $E$ into a circle with center $(0,0)$. The 
homeomorphism $h=h_{1} h_{2}$ takes each $M_{i}$ into a circle with center $(0,0)$; if $M$ is a proper subset of $C, h$ can be modified so as to take each $M_{i}$ into a horizontal interval.

\section{REFERENCES}

1. W. A. Blankinship, Generalization of a construction of Antoine, Ann. of Math. 53 (1951), 276-297.

2. Jo Ford, Imbedding collections of compact 0-dimensional subsets of $E^{2}$ in continuous collections of mututally exclusive arcs, Fund. Math 58 (1966), 45-57.

University of Georgia, Auburn University and UNIVERSITY OF MICHIGAN 